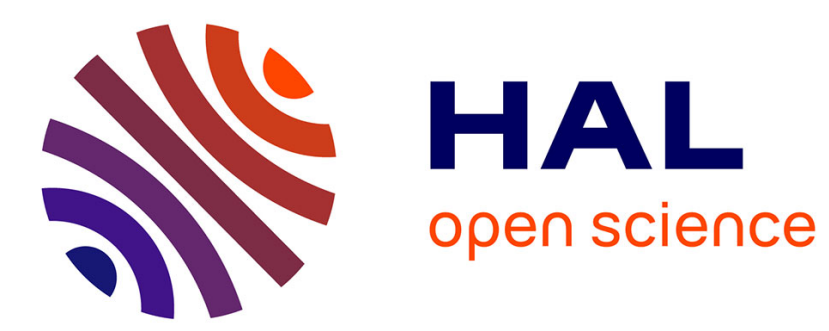

\title{
Deuil périnatal paternel et relation d'objet virtuelle
}

Marianne Dollander

\section{To cite this version:}

Marianne Dollander. Deuil périnatal paternel et relation d'objet virtuelle. Dialogue , 2014. hal02371262

\section{HAL Id: hal-02371262 \\ https://hal.science/hal-02371262}

Submitted on 19 Nov 2019

HAL is a multi-disciplinary open access archive for the deposit and dissemination of scientific research documents, whether they are published or not. The documents may come from teaching and research institutions in France or abroad, or from public or private research centers.
L'archive ouverte pluridisciplinaire HAL, est destinée au dépôt et à la diffusion de documents scientifiques de niveau recherche, publiés ou non, émanant des établissements d'enseignement et de recherche français ou étrangers, des laboratoires publics ou privés. 


\section{Deuil périnatal paternel et relation d'objet virtuelle}

\section{MARIANNE DOLLANDER ${ }^{1}$}

\section{RESUME}

L'objet de cet article est de proposer une conceptualisation du deuil périnatal chez l'homme. La première partie, générale, décrit brièvement la situation des parents face à ce type de mort, ainsi que les singularités du deuil périnatal au masculin.

La seconde partie développe plus finement la question de la symbolisation de la perte chez le père. Une réflexion sur le continuum narcissico-objectal de l'investissement du bébé in utero est engagée, qui mènera à une proposition de conceptualisation du deuil périnatal centrée sur la notion de Relation d'Objet Virtuelle (Missonnier, 2009).

Les histoires de messieurs B. et P. permettront d'illustrer ces propos.

\section{MOTS-CLES}

Deuil périnatal, paternité, relation d'objet virtuelle.

Les répercussions du deuil périnatal ont été étudiées à profusion chez la femme (Loraux, 1990 ; Rousseau, 1998 a ; Ferreri, 2003 ; Flis-Trèves, 2004 ; Martineau, 2008). Son impact sur la famille et l'entourage (Rousseau, 1995, 1998 b ; Mazet et Lebovici, 1996 ; Clerget, 2004 ; Coq, Romano, Scelles, 2011 ; Soubieux, 2011, 2013) et, dans une moindre mesure, sur le couple (Hébert, 1996), l'a été également. Mais en revanche très peu d'écrits ont concerné le deuil périnatal au masculin. C'est sur ce point que cet article ambitionne d'apporter sa pierre à l'édifice.

La «préoccupation primaire » (Boulet, 1997 ; Sirol, 2002) que développe le jeune père entraîne la nécessité d'un deuil en cas de décès périnatal. Un premier tour d'horizon nous

\footnotetext{
${ }^{1}$ Maître de Conférences en psychologie clinique, laboratoire Interpsy (équipe Interval) Université de Lorraine, site de Nancy, 3 place Godefroy-de-Bouillon, B.P. 3397, 54015 Nancy cedex. Psychologue clinicienne et psychanalyste. Marianne.Dollander@univ-lorraine.fr
} 
permettra de poser les particularités d'un tel deuil. Une seconde partie tentera de formaliser les modalités d'élaboration d'une telle perte. Concernant très spécifiquement la symbolisation de la perte, la conceptualisation de Missonnier (2009) en termes de relation d'objet virtuelle (ROV) se révélera particulièrement précieuse.

\section{Des pères et des mères face à la mort périnatale}

Le décès d'un enfant, est-il besoin de le dire, constitue un événement dramatique pour ses parents (Raimbault, 1996 a, 1996 b ; Bacqué, 1997 ; Hanus, 2001 ; Legros, 2001 ; Uren et coll., 2002 ; Soubieux, 2008, 2010, 2011; Glattigny-Dallay, 2011, 2013). Tous les cliniciens s'accordent à considérer ce type de décès comme celui où la perte d'objet est à son comble (de Broca, 1997). Perte d'objet, car lorsque le projet d'enfant est investi, une relation d'objet se construit dès avant la naissance entre le «devenant parent» et l'«enfant du dedans» (Missonnier, 2009).

Terrible paradoxe, la mort périnatale représente l'irruption de la mort là où la vie devait advenir. La succession des générations scande le temps familial, construit l'arbre généalogique sur lequel chacun pourra se situer lui-même et placer ses ascendants et descendants. Or la mort périnatale vient bousculer tous ces repères, chez les parents en deuil, mais également dans la perception que leur entourage se fait d'eux : «ils ne sont plus un couple sans enfant comme auparavant, ils ne sont pas vraiment reconnus comme parents, ils ne sont plus vraiment seulement fils ou fille de leurs parents » Soubieux (2008, p.29). Les parents en deuil doivent en outre affronter une blessure narcissique intolérable, puisque leur enfant emporte avec lui une part de leur propre narcissisme, conjointement à tous les espoirs et idéaux qu'ils avaient placés en lui.

Famille bouleversée, mère et père en deuil, nous nous concentrerons dans le point suivant sur les singularités du deuil périnatal au masculin. 


\section{Les singularités du deuil périnatal au masculin}

Caractéristique particulièrement cruelle et inductrice de confusion, le père endeuillé doit tout à la fois élaborer son nouveau statut de père - et en faire le deuil - et renoncer à l'enfant qui l'a fait père. Monsieur B. ${ }^{2}$ parle ainsi du jour où sa fille est née et décédée : «Malheureusement le 8 juin je me suis senti père même si elle était perdue... [...] c'est à partir de ce moment-là j'ai employé le mot père... » Cette problématique est sous doute plus aiguë chez les primipères, au sens où le foyer reste vide d'un enfant qui aurait appelé et permis l'exercice de cette parentalité. L'unique changement de statut généalogique manque à faire sens pour ces hommes « désenfantés », auxquels échappe la paternité incarnée, celle des relations quotidiennes.

On comprend que le deuil soit d'autant plus malaisé qu'il survient avant où au moment de la naissance, avant que le père ait pu faire connaissance avec un enfant vivant dont il ait pu se construire une représentation. Ainsi que l'exprime monsieur B. : «Le fait de l'avoir perdue là subitement, bah on se retrouve un petit peu vide [...], je la sentais bouger mais... visualiser à part les échographies euh... voilà c'était quand même un peu abstrait quoi. »

Cela dit, le devenant père n'est pas démuni de toute représentation psychique de son enfant. D'une part, certains éléments du suivi de la grossesse (échographies, haptonomie...) aideront à la construction de représentations concrètes et d'éprouvés sensoriels, via le toucher (Soulé, Gourand, Missonnier, Soubieux, 1999 ; Rossigneux-Delage, 2004 ; Soubieux, 2008). D'autre part, le devenant père porte psychiquement, lui aussi, un enfant imaginaire et réalise un travail psychique reposant sur la mise en mots et la mise en récit (Sirol, 2002). J'ajouterai que cette mise en mots permettra l'élaboration du deuil périnatal, puisque le travail de symbolisation de la perte s'étayera sur le processus engagé dès les premiers temps de la grossesse.

Ces considérations appellent une réflexion plus approfondie sur le processus intrapsychique de symbolisation de la perte périnatale chez le père. Nous développerons ces propos théoriques en nous étayant sur les histoires de messieurs P. et B. ${ }^{3}$. Monsieur P., âgé de 30 ans au jour de notre rencontre, a perdu son bébé un an auparavant, suite à la fausse-couche tardive de sa compagne, à 21 semaines d'aménorrhée. Dirigé vers une psychologue dès l'événement, le couple a pu bénéficier d'une écoute qui a aidé monsieur P. à avancer constructivement dans son deuil.

\footnotetext{
${ }^{2}$ Nous présenterons plus longuement monsieur B. dans la suite de cet article.

${ }^{3}$ Claire GRANDGIRARD a rencontré messieurs B. et P. au cours de son TER de Master 1 de psychologie, réalisé sous ma direction (Grandgirard, 2012). Je la remercie de m'avoir confié ces données. Les deux hommes ont également donné leur consentement à l'exposé de leur témoignage dans le cadre d'une publication.
} 
Monsieur B. est âgé de 46 ans au moment où nous le rencontrons. Le décès de leur premier enfant, une petite fille qu'ils avaient prénommée «Zoé » ${ }^{4}$, s'était produit déjà 14 ans auparavant, dans des circonstances particulièrement traumatiques pour le couple. L'accouchement s'était déclenché prématurément et, le fœus n'étant pas viable, les soignants avaient procédé à une fœeticide in utero. Monsieur B. nous apparaît malheureusement englué dans un deuil compliqué, comme en témoigne son discours lorsqu'on lui demande ce qu'il ressent aujourd'hui à propos de «Zoé » : «C'est un coup de poignard, pour moi c'est toujours... [...] le 8 juin 18h00 pour moi c'est... c'est ancré dans la tête... »

Le deuil en voie d'accomplissement de monsieur P. nous permettra de montrer l'implication de la relation d'objet virtuelle dans l'élaboration de la perte périnatale paternelle. Quant à l'histoire de monsieur B., elle fournira un contrepoint utile à notre réflexion.

\section{Le deuil périnatal paternel}

Le travail de deuil est généralement décrit dans la littérature comme l'accomplissement de trois phases (Bacqué, 1992 ; Hanus, 1994 ; Bacqué, Hanus, 2000), que nous rappelons ici succinctement : la première phase du travail de deuil, ou plus exactement sa phase préliminaire, est l'état de choc, provoqué par la nouvelle de la mort de l'être cher. Le travail de deuil proprement dit ne commence réellement que lorsque le temps du déni et du refus est dépassé, et que s'installe la souffrance dépressive. C'est là que s'effectue le désinvestissement de l'objet, qui constitue l'essence du travail de deuil. Enfin, la phase d'adaptation (Bacqué, 1992), ou de terminaison (Hanus, 1994, 2000), est marquée par l'investissement de nouveaux objets et la création de nouveaux liens.

\section{La symbolisation de la perte}

La symbolisation de la perte permet d'établir un lien entre la réalité extérieure et notre monde interne, grâce à deux processus contemporains et complémentaires (Dollander, 1999). Le

\footnotetext{
Pseudonyme.
} 
premier correspond à la symbolisation de l'objet perdu : il s'agit de mettre en représentation et d'intégrer à notre monde interne cet objet et la relation que l'on entretenait avec lui. L'objet intériorisé n'est alors plus vécu comme un équivalent de l'objet externe, et le rapport à ce dernier est dorénavant marqué du sceau de la perte. Notons que cette symbolisation nécessite d'élaborer une représentation entière de l'objet perdu, intégrant ses côtés positifs et négatifs.

Le travail de deuil implique également la symbolisation des affects liés à la perte (déchirement, souffrance, peur, détresse, sentiment d'abandon, colère, culpabilité...), autrement dit la possibilité de les lier entre eux ainsi qu'aux représentations les ayant suscités, sans qu'une quantité d'affects excessive ne vienne paralyser le travail de la pensée. Cette symbolisation est d'autant plus indispensable qu'il n'est possible de parvenir à une représentation stable de l'objet que si les affects lui étant attachés sont suffisamment élaborés.

Ceci posé, il est indubitable que le deuil périnatal n'est pas un deuil comme les autres, du fait du manque de différenciation de l'objet et de la force avec laquelle il était investi. L'enfant non né n'est pas un objet dont les représentations s'élaborent psychiquement de la même manière que lorsque l'être cher disparu est un adulte, ou même un enfant plus grand. Perdre un enfant représente une amputation, quel que soit l'âge auquel intervient le décès, mais à cela s'ajoute, dans les morts in utero ou très précoces, le fait que les parents restent devant un vide particulièrement cruel en termes d'images ou de souvenirs d'une vie partagée: pas de photographies, pas d'anniversaires en famille, pas de rires qui résonnent à l'oreille... Comme en témoigne monsieur B., parlant de sa participation à des groupes de parents endeuillés : « Le souci qu'on rencontre c'est que nous on n'a rien partagé [...]. Leur dire quoi ? Leur parler de néant, leur parler d'espoirs, leur parler de rêves... c'est extrêmement abstrait... »

Autrement dit, peu de traces pour donner une consistance externe à cet être auquel il faut désormais renoncer. Les émotions sont d'une violence extrême : l'horreur de se sentir arracher la chair de sa chair, la culpabilité d'avoir failli à son devoir de protection parental, la peur de devenir fou de douleur. La violence de l'ambivalence (la colère contre le fœus se heurte à des sentiments d'injustice, de culpabilité et de honte) est particulièrement terrible à éprouver (Soubieux, 2008).

Si l'on revient à la question centrale de la symbolisation de l'objet, le fait que l'investissement objectal d'un être tout juste ou non encore né soit éminemment immature, complique la création d'une représentation de l'objet perdu marquée du sceau de la perte. 
L'hémorragie narcissique menace toujours davantage dans les situations de deuil d'enfant et, qui plus est, de deuil périnatal, du fait de la massivité de l'investissement narcissique parental.

L'ensemble de ces réflexions tend à faire penser que le deuil périnatal sera d'autant plus élaborable (car l'objet perdu sera identifiable et «consistant» psychiquement et car l'identification à l'objet perdu sera moindre) que l'investissement objectal sera avancé. Comprendre le deuil périnatal nécessite donc de se pencher sur la construction et la maturation progressive de l'investissement de l'« enfant du dedans » (Missonnier, 2009), que l'on peut décrire comme suivant un continuum narcissico-objectal (Sirol, 2002 ; Missonnier, 2009).

\section{Le continuum narcissico-objectal de l'investissement du bébé in utero}

\section{Massivité du déplacement narcissique}

Lorsque le projet d'enfant est investi, l'enfant à venir est l'objet d'un déplacement narcissique massif de la part de son père. L'amour paternel constitue, dans les termes de S. Freud (1914), une métamorphose en amour d'objet du narcissisme du nouveau père. Tout cela pose les bases à l'adoption psychique, que l'on peut définir comme l'investissement narcissique et objectal de l'enfant, indispensable car fondateur du lien qui se développera après la naissance (Guyotat, 1995 ; Aubert-Godard, 1999). Deux auteurs (Bayle, 2003, 2005, 2009 ; Missonnier, 2004, 2006, 2009) soulignent plus particulièrement que cette adaptation se noue dès la vie intrautérine.

\section{Relation d'Objet Virtuelle et construction d'un investissement (pré)objectal}

Contrairement au point de vue psychanalytique le plus commun, qui fait du fotus une incarnation du narcissisme parental, Missonnier (2004) considère ce tableau comme un point de départ. D'après lui, c'est au cours de la grossesse que se joue le passage d'un «investissement narcissique extrême (qui tend vers un degré zéro de l'objectal) à l'émergence progressive d'un investissement (pré)objectal » (Missonnier, 2009, p.132), tout cela étant permis par l'effet du virtuel. Monsieur P. témoigne bien de la progressivité de l'investissement : « Je savais que j'allais 
être père quand j'ai appris qu'on allait avoir un bébé [...] et je suis devenu parent quand on l'a perdue... je sais pas comment dire... ça se transforme, bien qu'on l'ait pas eu vivante et qu'on n'ait pas pu l'élever. »

S'étayant sur la définition de Diatkine (1995), qui voit dans le travail du virtuel l'actualisation d'un potentiel créatif, Missonnier (2009, p.160) souligne que c'est ce même travail qui «aboutit progressivement à une authentique anticipation de l'altérité objectale de l'enfant à venir ». Ce qu'il appelle la Relation d'Objet Virtuelle (ROV) se construit au sein de ce processus. Monsieur P. l'illustre fort joliment : «Je lui parlais déjà, je savais pas si c'était un garçon ou une fille et c'était déjà mon enfant, je lui parlais dans le ventre ». Il souligne à quel point il attribuait une subjectivité en construction à son enfant, dès sa conception : «je pense qu'on doit compter l'âge d'un enfant à partir des 9 mois [in utero] où il grandit... ».

Faure-Pragier $(2003,1995)$ décrit l'importance du lien à l'autre dans le processus virtuel. D'après elle, «le virtuel fonctionne entre réalité matérielle et réalité psychique, entre soi et l'autre, dans l'espace transitionnel décrit par Winnicott» (Pragier, Faure-Pragier, 1995, p.78). Dans cet esprit, Missonnier (2009) définit la ROV comme la constitution du lien réciproque biopsychique s'établissant au cours de la grossesse entre les devenant parents et l'enfant du dedans : les premiers opèrent une «nidification » biopsychique, alors que le second s'inscrit dans une « nidation » biopsychique (Missonnier, 2006).

Cette nidation et cette nidification sont contenues dans un « espace virtuel » que l'on peut rapprocher du concept d'espace transitionnel, décrit par Winnicott (1971) comme une aire intermédiaire entre l'enfant et sa mère. C'est dans cet espace virtuel, cohabité à trois, que s'inscrit la relation d'objet virtuelle. Laissons la parole à monsieur B. : «L'enfant on le conçoit à deux... [...] Je voyais son ventre grossir, je la sentais bouger, je sentais que quand je mettais ma main elle... ça se rapprochait... elle était abstraite mais présente... Je vivais ' Zoé'” par sa maman. »

Le concept d'enfant imaginaire (Lebovici, 1983, 1994 ; Soulé, 1982) fut proposé il y a déjà longtemps pour décrire l'enfant porté dans les désirs et les fantasmes de ses parents. L'intérêt du concept d'objet virtuel est de souligner encore davantage à quel point l'«être conçu » (Bayle, 2003, 2005, 2009) modèle également, dans la réciprocité, le fonctionnement psychique de ses parents. J'ajouterai que l'enfant virtuel est envisagé dans une dynamique triadique, et non dyadique. Ce concept est en outre particulièrement dynamique, puisqu'il met en exergue le côté évolutif de ce processus : l'enfant virtuel n'est pas statique, mais ne sera perçu et reconnu par ses 
parents comme possédant une véritable altérité qu'au prix d'un travail aléatoire et individuel de chacun d'eux (Missonnier, 2004, 2006, 2009).

Dans le contexte du deuil périnatal, la question aiguë est la suivante : comment conceptualiser le désinvestissement de l'objet externe au profit de l'objet interne, lorsque l'objet perdu ne fut jamais (ou presque) vivant qu'à l'intérieur du corps de sa mère, étant donné que l'on ne peut faire le deuil que d'un objet investi et ayant trouvé une forme de représentation intrapsychique sous forme d'objet interne?

Symboliser l'objet perdu, c'est aussi intérioriser la relation qui nous liait à lui. Ainsi que le questionne Hanus (2001, p.8), «quelle est la relation avec un tout-petit avant sa naissance ? [...] Lorsque l'enfant meurt in utero, ses singularités personnelles objectives sont encore restreintes et la confrontation avec l'enfant du désir n'est pas simple. » Or le concept de relation d'objet virtuelle permet précisément de décrire les prémices d'une relation d'objet différenciée, en construction tout au long de la grossesse. Il nous permet donc de comprendre à quel objet et à quelle relation il s'agit de renoncer, d'identifier les voies d'élaboration de ce deuil.

\section{Proposition de conceptualisation du deuil périnatal grâce à la notion de Relation d'Objet Virtuelle}

\section{Deuil périnatal : renoncement à l'objet virtuel}

Concernant le lien à l'objet, l'accomplissement satisfaisant du travail de deuil nécessite deux mouvements : le désinvestissement de l'objet réel, allant de pair avec une intériorisation d'ordre symbolique. En cas de deuil périnatal, l'objet d'amour appelé au désinvestissement est l'objet virtuel. Nous savons à quel point la différenciation d'avec l'objet perdu est fondamentale à l'élaboration du deuil. Les modalités de la ROV imprimeront donc nécessairement leur marque sur l'avancée de la symbolisation de la perte puisqu'elles détermineront l'évolution de la différenciation au cours de la grossesse, de l'«anticipation adaptative au fil des jours de l'altérité de l'enfant virtuel » (Missonnier, 2009, p.201-202). 


\section{Lien prénatal père-enfant}

Archaïque s'il en est, la ROV est une relation d'objet utérine, antérieure aux relations d'objets orale, anale puis génitale classiquement décrites par la psychanalyse. Cette relation d'objet utérine «représente la matrice de toute la filière ultérieure de la relation d'objet » (Missonnier, 2009, p.132).

Le terme de «virtuel » n'est pas à opposer à « réel » mais à «actuel»: autrement dit, la rencontre intersubjective «virtuelle » n'a pas encore eu lieu au sens de l'accompli, mais existe déjà en tant que potentialité. Monsieur B. parle clairement de ses futures relations avec son bébé, facilitées d'après lui par le fait qu'il s'agisse d'une fille. «Je ne veux pas dire que je l'imaginais comme une princesse, mais c'est vrai que moi c'était quelque chose de magnifique... en plus une fille !! [...] Je suis beaucoup plus à l'aise avec la gente féminine. »

Cette relation d'objet, virtuelle au cours de la grossesse, devra donc s'actualiser à partir de la naissance. Ce concept permet de rendre compte de la relation telle qu'elle existe entre l'endeuillé et pour le petit être décédé, au moment où survient le drame, c'est-à-dire l'époque prénatale.

Missonnier (2009) souligne que, mutatis mutandis, l'investissement paternel de l'enfant du dedans évolue au cours de la période prénatale en fonction des modalités d'une « transparence psychique » (terme introduit par Bydlowski, 1991) dont l'homme fait lui aussi l'expérience. Cette relation d'objet virtuelle concerne «l'ensemble des comportements, des affects et des représentations (conscientes, préconscientes et inconscientes) à l'égard de l'embryon, puis du fotus » (Missonnier, 2009, p.131). Ainsi comprend-on sur quels matériaux psychiques l'homme pourra s'étayer pour construire des représentations favorisant la symbolisation de la perte, pour donner corps à une représentation de l'objet perdu.

\section{Donner corps à une représentation de l'objet perdu}

Un autre intérêt du concept d'objet virtuel, dans le cadre du deuil périnatal, est donc de donner consistance à un objet non uniquement imaginaire, mais appelé à être accueilli comme un objet réel. La ROV, au sein de laquelle niche l'enfant virtuel, constitue un processus continu d'investissement psychique. Dynamique et adaptative, elle permet de construire une représentation de l'objet potentiel. 
L'histoire de monsieur B. prouve dramatiquement à quel point le deuil peut être entravé lorsque cette représentation de l'objet potentiel est pulvérisée par un réel traumatique : La petite «Zoé » étant décédée à cinq mois et demi de gestation, son corps fut en effet déposé dans la « poubelle anatomique », ce dont monsieur B. est parfaitement conscient, du fait de sa profession d'infirmier : «Elle a été mise avec la poubelle "anatomique"... avec les membres sectionnés, avec l'amputation, avec les bouts d'intestin, avec un bout de... » Tout cela est d'autant plus cruel pour lui que « à 10 jours près... elle aurait pu avoir une sépulture $»^{5}$.

On ne peut faire le deuil que d'un enfant dont l'existence s'est inscrite psychiquement, d'un objet bénéficiant d'une représentation interne. A ce titre, la reconnaissance légale et l'inscription de l'enfant dans la filiation sont des facteurs importants dans un tel deuil. Ainsi, monsieur P. et sa compagne, qui vivaient jusqu'alors en union libre, ont-ils ressenti le besoin de poser un acte légal : «On s'est pacsés et elle apparaît sur notre livret de famille comme premier enfant né décédé[...]. C'était pour officialiser la famille bien qu'on n'ait pas notre fille... ça a quand même créé une famille. »

\section{Ouverture}

L'intérêt des concepts de ROV et d'enfant virtuel est de renforcer la consistance théorique d'une dimension que les cliniciens qui prennent en charge les parents en deuil connaissent bien : l'enfant non (ou à peine) né peut sembler éthéré - et semble d'ailleurs hors de portée de bien des membres de l'entourage -, mais sa réalité psychique est ô combien massive.

Penser le deuil périnatal en termes de renoncement à l'objet virtuel signifie concrètement que le père pourra se saisir de ses émotions et représentations anticipées concernant le devenir de l'enfant, du lien qu'il aurait souhaité entretenir avec lui, de la vie qu'il imaginait pour lui-même, l'enfant et sa mère, et les frères et sœurs déjà présents ou à venir. Comme l'explicite monsieur P. : «Maintenant je me dis ah j'aurai peut-être fait ça avec "Julie"6, elle aurait eu un an... [...] et régulièrement je me dis ah là elle aurait eu tel âge et elle aurait peut-être fait ça et ça... »Ces

\footnotetext{
${ }^{5}$ Loi no ${ }^{\circ}$ 93-22 du 8 janvier 1993 : L'existence juridique est reconnue à un enfant, soit né mort à au moins 180 jours de vie in utero, soit né vivant à partir de 22 semaines d'aménorrhée ou 500 grammes.
}

Pseudonyme. 
scénarios imaginaires et envisagés pour le futur seront, comme des souvenirs, «mis sur le métier » (Freud, 1915) et désinvestis pour être frappés du sceau de la perte. Legros (2001) précise pour sa part que ces deuils particuliers gardent une part d'inconsolable, et qu'un deuil fait n'est pas forcément un deuil « clos ».

Sous forme de dernière remarque, un paradoxe reste à souligner : plus la grossesse avance, plus l'investissement, l'amour et les espoirs logés en l'enfant grandissent (ce qui est de nature à amplifier la souffrance du déchirement), mais aussi plus la différenciation d'avec l'objet virtuel se structure (ce qui est fondamental à l'élaboration du deuil).

\section{Bibliographie}

AUBERT-GODARD, A. 1999. «Devenir père, un risque narcissique ? », dans M. Dugnat (sous la direction de), Devenir père, devenir mère, Ramonville Sainte-Agne, Erès, 129-142.

BACQUÉ, M.-F. 1992. Le deuil à vivre, Paris, Odile Jacob.

BACQUÉ, M.-F. 1997. Deuil et santé, Paris, Odile Jacob.

BACQUE, M.-F., HANUS, M. 2000. Le deuil, Paris, Puf, coll. « Que sais-je ? ».

BAYLE, B. 2003. L'embryon sur le divan. Psychopathologie de la conception humaine, Paris, Elvesier Masson.

BAYLE, B. 2005. L’enfant à naître, Toulouse, Erès.

BAYLE, B. 2009. «Comprendre la conception humaine. Présentation du groupe "Psychopathologie et développement anténatal" de la Société Marcé (Chartres)», dans C. Morrisseau, C (sous la direction de), Lorsque la parentalité paraît, Paris, Puf, 95-100.

BOULET, F.-X. 1997. «Un père attend un enfant », Spirale, 6, Erès, 87-92.

de BROCA, A. 1997. Deuils et endeuillés, (se) comprendre pour mieux (s')écouter et (s')accompagner, Paris, Masson.

BYDLOWSKI, M. 1991. «La transparence psychique de la grossesse », Etudes freudiennes, 32, Etudes freudiennes, 135-142.

CLERGET, J. (Sous la direction de). 2004. «Mort d'un bébé, deuil périnatal. Témoignages et réflexions », Spirale, 31, Erès, 13-130.

COQ, J.-M. ; ROMANO, J. ; SCELLES, R. 2011. La mort d'un enfant : processus de deuil dans le groupe familial, Neuropsychiatrie de l'enfance et de l'adolescence, 59, Elvesier Masson, 356361. 
CUPA, D. 2007. «Le complexe de grossesse du père », dans S. Missonnier ; B. Golse ; M. Soulé (sous la direction de), La grossesse, l'enfant virtuel et la parentalité, Paris, Puf, 161-184.

DOLLANDER, M. 1999. Figures de l'absence. Contribution à la clinique du travail de deuil, Thèse de doctorat en psychologie, Université Nancy 2, octobre 1999. Lille, Editions du Septentrion, 2001.

FAURE-PRAGIER S. 2003. Le virtuel pourquoi ça marche ? Hypothèses psychanalytiques, dans S. Missonnier ; H. Lisandre (sous la direction de) Le virtuel : la présence de l'absent, Paris, EDK, 41-58.

FERRERI, F. 2003. «La dépression de deuil périnatal », dans F. Ferreri, La dépression au féminin, Montrouge, John Libbey Eurotext.

FLIS-TREVES, M. 2004. Le deuil de maternité, Paris, Calmann-Lévy.

FREUD, S. 1914. «Pour introduire le narcissisme », dans La vie sexuelle, Paris, PUF, 1969, 81105.

FREUD, S. 1915. «Deuil et mélancolie », dans Métapsychologie, trad. fr. J. Laplanche et J.-B. Pontalis, Paris, Gallimard, 1968.

GAUTHIER, Y., LEBOVICI, S.; MAZET, P., VISIER, J. (Sous la direction de). 1993. Tragédies à l'aube de la vie. Répercussions sur les familles. Paris, Bayard Editions, Inserm CTNERHI.

GLATIGNY-DALLAY, E. 2011. «Le deuil périnatal : un deuil particulièrement difficile pour tous », Confrontations psychiatriques, 50, Editions médicales / Specia, 200-224.

GLATTIGNY-DALLAY, E. 2013. «Le deuil périnatal de "l'enfant né sans vie" », Annales médico-psychologiques, 171, 3, Elvesier Masson, 182-188.

GRANDGIRARD, C. 2012. Des hommes face à la perte prénatale : le deuil de l'objet virtuel, TER de M1 de psychologie, Université de Lorraine.

GUYOTAT, J. 1995. Filiation et puerpéralité, logiques du lien, Paris, Puf.

HANUS, M. 1994. Les deuils dans la vie, Paris, Maloine.

HANUS, M. 2000. La mort retrouvée, Paris, Frison-Roche.

HANUS, M. 2001. «Le deuil de ces tout-petits enfants », Etudes sur la mort, 119, L'esprit du temps, 7-15.

HEBERT, M. 1996. «Retentissement sur le couple», dans P. Mazet, S. Lebovici (sous la direction de), Mort subite du nourrisson, un deuil impossible ?, Paris, Puf, 227-232.

LEBOVICI, S. 1983. Le nourrisson, la mère et le psychanalyste, Paris, Païdos/Le Centurion. 
LEBOVICI, S. 1994. «Les interactions fantasmatiques », Revue de Médecine Psychosomatique, 37/38, La pensée sauvage, 39-50.

LEGROS, J-P. 2001. «L'arrêt de vie in utero ou l'errance des fœtus ; un possible deuil », Etudes sur la mort, 119, L'esprit du temps, 63-75.

LORAUX, N. 1990. Les mères en deuil, Paris, Le Seuil.

MARTINEAU, E. 2008. Surmonter la mort d'un enfant attendu. Dialogue autour du deuil périnatal, Lyon, Chronique sociale.

MAZET, P. ; LEBOVICI, S. (Sous la direction de), 1996. Mort subite du nourrisson, un deuil impossible ?, Paris, Puf.

MISSONNIER, S. 2004. "L'enfant du dedeans et la relation d'objet virtuelle », dans S. Missonnier, B. Golse, M. Soulé (sous la direction de). La grossesse, l'enfant virtuel et la parentalité, Paris, Puf, 119-144.

MISSONNIER, M. 2006. « Nidification fœtale, nidation parentale », dans J. Bergeret, M. Soulé, B. Golse (sous la direction de), Anthropologie du fœtus, Paris, Dunod, 83-98.

MISSONNIER, S. 2007. «Le premier chapitre de la vie. Nidification parentale. Nidation fœtale », La psychiatrie de l'enfant, L 1, Puf, 61-80.

MISSONNIER, S. 2009. Devenir parent, naître humain. La diagonale du virtuel. Paris, Puf.

PRAGIER, G. ; FAURE-PRAGIER S. 1995. « Au-delà du principe de réalité : le virtuel », Revue française de psychanalyse, LIX, 1, Presses Universitaires de France, 63-84.

RAIMBAULT, G. 1996 a. «Des parents devant la mort de leur enfant », Psychiatries, 117, Association française de psychiatres d'exercice privé, 31-38.

RAIMBAULT, G. 1996 b. Lorsque l'enfant disparaît, Paris, Odile Jacob.

ROUSSEAU, P. 1995. «Les pertes périnatales, la famille, les soignants et la société », Devenir, 7, 1, Médecine \& Hygiène, 31-60.

ROUSSEAU, P. 1998 a. «Une grossesse après l'autre dans le deuil périnatal », Spirale, 8, Erès, 53-65.

ROUSSEAU, P. 1998 b. «Deuil périnatal, abord transgénérationnel », Devenir, 10, 3, Médecine \& Hygiène, 35-65.

ROSSIGNEUX-DELAGE, P. 2004. «Grossesse et haptonomie », dans S. Missonnier, B. Golse, M. Soulé (sous la direction de), La grossesse, l'enfant virtuel et la parentalité. Éléments de psycho(patho)logie périnatale, Paris, Puf, 223-253. 
SIROL, F. 2002. «Interruption du processus de paternité », dans C. Guillaumont (sous la direction de), Les troubles psychiques précoces du post-partum, Ramonville Saint-Agne, Erès, 107-116.

SOUBIEUX, M.-J. 2008. Le berceau vide. Deuil périnatal et travail du psychanalyste. Toulouse, Erès.

SOUBIEUX, M.-J. 2010. Le deuil périnatal, Paris, Fabert.

SOUBIEUX, M.-J. 2011. Le bébé des limbes, Carnet psy, 154 : 42-46.

SOULE, M. 1982. «L'enfant dans la tête, l'enfant imaginaire », dans La dynamique du nourrisson, Paris, ESF, 135-175.

SOULE, M. ; GOURAND, L. ; MISSONNIER, S. ; SOUBIEUX, M.-J. 1999. Ecoute voir... l'échographie de la grossesse. Les enjeux de la relation. Ramonville Saint-Agne, Erès, coll. «A l'aube de la vie ».

UREN, T. H. ; WASTELLE, C. A. 2002. «Attachment and meaning-making in perinatal bereavement », Death Studies, 26, Routledge, 279-308.

WINNICOTT, D.W. 1971. Jeu et réalité. L'espace potentiel, Paris, Gallimard, coll. «Connaissance de l'inconscient », 1975.

\section{AbStract}

This article proposes a conceptualisation of men's perinatal loss. The first part is general and provides a brief account of the situation of parents when confronted with perinatal death. It also focuses on the specifics of perinatal bereavement for men.

The second part explores the question of fathers' symbolic representation of loss. It examines their narcissistic objectal investment of the baby in utero. This will lead to a proposed conceptualization of perinatal loss, that will focus on the notion of Virtual Object Relationship (Missonnier, 2009 a).

This point is illustrated by Mr B.'s and Mr P.'s personal stories.

\section{KEY-WORDS}

Perinatal loss, Paternity, Virtual Object Relationship. 